\title{
Robust Adaptive Sliding Mode Control for Generalized Function Projective Synchronization of Different Chaotic Systems with Unknown Parameters
}

\author{
Xiuchun Li, ${ }^{1}$ Jianhua $\mathrm{Gu},{ }^{1}$ and Wei $\mathrm{Xu}^{2}$ \\ ${ }^{1}$ Centre for High Performance Computing, Northwestern Polytechnical University, Xian 710072, China \\ ${ }^{2}$ Department of Applied Mathematics, Northwestern Polytechnical University, Xian 710072, China \\ Correspondence should be addressed to Xiuchun Li; lixiuchun@nwpu.edu.cn
}

Received 26 April 2013; Revised 16 July 2013; Accepted 16 July 2013

Academic Editor: Ningsu Luo

Copyright ( 2013 Xiuchun Li et al. This is an open access article distributed under the Creative Commons Attribution License, which permits unrestricted use, distribution, and reproduction in any medium, provided the original work is properly cited.

\begin{abstract}
When the parameters of both drive and response systems are all unknown, an adaptive sliding mode controller, strongly robust to exotic perturbations, is designed for realizing generalized function projective synchronization. Sliding mode surface is given and the controlled system is asymptotically stable on this surface with the passage of time. Based on the adaptation laws and Lyapunov stability theory, an adaptive sliding controller is designed to ensure the occurrence of the sliding motion. Finally, numerical simulations are presented to verify the effectiveness and robustness of the proposed method even when both drive and response systems are perturbed with external disturbances.
\end{abstract}

\section{Introduction}

Chaos synchronization has been a hot topic since the pioneering work of Pecora and Carroll [1]. Various methods and techniques have been proposed for the control and synchronization of chaotic systems. In the past, most synchronizing techniques, such as linear and nonlinear feedback control [2$4]$, adaptive control $[5,6]$, sliding mode control $[7,8]$, and the liner matrix inequality (LMI) technique [9], have been designed for realizing synchronization of chaotic system.

Amongst all kinds of chaos synchronization, projective synchronization (PS), which was first reported by Mainieri and Rehacek [10], has been extensively investigated in the recent years because it can obtain faster communication with its proportional feature. this means that the drive and response systems can synchronize up to a scaling factor. Subsequently, Chen et al. [11] put forward another new projective synchronization, which is called function projective synchronization (FPS). Function projective synchronization is that the chaotic signals of the drive and response systems can synchronize up to a scaling function factor. Recently, some researchers [12, 13] have proposed a new synchronization scheme, called generalized function projective synchronization (GFPS), which is an extension of FPS. GFPS is more generalized and can give information transmission more security in secure communication. Wang and Fan [12] and Li and Zhao [13] have, respectively, used the state observer and adaptive controller to realize GFPS of a class of chaotic systems. It is a pity that they have not considered the influence of external perturbations.

In practice, the uncertainties and external disturbances affect the whole dynamics of the systems. So Yang and $\mathrm{Ou}$ [14] have discussed complete synchronization and antisynchronization of chaotic gyros with external perturbations using adaptive sliding mode control. But this method is not universal due to the discussion for specific chaotic system. Xiang and Chen [15] and Aghababa and Akbari [16] have all proposed sliding mode control method to synchronize two different chaotic systems with disturbances. Unfortunately, they $[15,16]$ only realize complete synchronization, and the parameters of the system in [14-16] are all known.

So, in this paper, we will extend adaptive sliding mode control method to realize GFPS of a class of chaotic systems with unknown parameters. According to two major stages of sliding mode design, we firstly give sliding mode surface, 
and the controlled system is robust to external perturbations on this surface. Secondly, adaptive sliding mode controller and the update laws of unknown parameters are given in order to make the system reach and subsequently stay on the sliding surface from any initial values. Finally two different chaotic systems are illustrated to verify the effectiveness of the proposed method, and it can be found that the designed controller has stronger robustness when both drive and response systems are all perturbed with external disturbances.

\section{Description of Chaotic System}

Consider a class of chaotic systems with unknown parameters which can be described as

$$
\begin{aligned}
\dot{x}_{1}= & F_{1}\left(x_{1}, x_{2}, \ldots, x_{n}\right)+f_{11}\left(x_{1}, x_{2}, \ldots, x_{n}\right) p_{11} \\
& +f_{12}\left(x_{1}, x_{2}, \ldots, x_{n}\right) p_{12} \\
& +\cdots+f_{1 m}\left(x_{1}, x_{2}, \ldots, x_{n}\right) p_{1 m}, \\
\dot{x}_{2}= & F_{2}\left(x_{1}, x_{2}, \ldots, x_{n}\right)+f_{21}\left(x_{1}, x_{2}, \ldots, x_{n}\right) p_{21} \\
& +f_{22}\left(x_{1}, x_{2}, \ldots, x_{n}\right) p_{22} \\
& +\cdots+f_{2 m}\left(x_{1}, x_{2}, \ldots, x_{n}\right) p_{2 m}, \\
& \vdots \\
\dot{x}_{n}= & F_{n}\left(x_{1}, x_{2}, \ldots, x_{n}\right)+f_{n 1}\left(x_{1}, x_{2}, \ldots, x_{n}\right) p_{n 1} \\
& +f_{n 2}\left(x_{1}, x_{2}, \ldots, x_{n}\right) p_{n 2} \\
& +\cdots+f_{n m}\left(x_{1}, x_{2}, \ldots, x_{n}\right) p_{n m},
\end{aligned}
$$

where $x=\left(x_{1}, x_{2}, \ldots, x_{n}\right)^{T} \in R^{n}$ denotes the state vector, $F_{i}, f_{i j}(i=1,2, \ldots, n ; j=1,2, \ldots, m)$ are nonlinear functions, and $p_{i j}(i=1,2, \ldots, n ; j=1,2, \ldots, m)$ are unknown parameters.

Take system (1) as the drive system; the response system, different from the system (1), with a controller $u=$ $\left(u_{1}, u_{2}, \ldots, u_{n}\right)^{T} \in R^{n}$, is as follows:

$$
\begin{aligned}
\dot{y}_{1}= & G_{1}\left(y_{1}, y_{2}, \ldots, y_{n}\right)+g_{11}\left(y_{1}, y_{2}, \ldots, y_{n}\right) q_{11} \\
& +g_{12}\left(y_{1}, y_{2}, \ldots, y_{n}\right) q_{12}+\cdots+g_{1 k}\left(y_{1}, y_{2}, \ldots, y_{n}\right) q_{1 k} \\
& +u_{1}, \\
\dot{y}_{2}= & G_{2}\left(y_{1}, y_{2}, \ldots, y_{n}\right)+g_{21}\left(y_{1}, y_{2}, \ldots, y_{n}\right) q_{21} \\
& +g_{22}\left(y_{1}, y_{2}, \ldots, y_{n}\right) q_{22}+\cdots+g_{2 k}\left(y_{1}, y_{2}, \ldots, y_{n}\right) q_{2 k} \\
& +u_{2}, \\
\vdots & \\
\dot{y}_{n}= & G_{n}\left(y_{1}, y_{2}, \ldots, y_{n}\right)+g_{n 1}\left(y_{1}, y_{2}, \ldots, y_{n}\right) q_{n 1} \\
& +g_{n 2}\left(y_{1}, y_{2}, \ldots, y_{n}\right) q_{n 2}+\ldots+g_{n k}\left(y_{1}, y_{2}, \ldots, y_{n}\right) q_{n k} \\
& +u_{n},
\end{aligned}
$$

where $y=\left(y_{1}, y_{2}, \ldots, y_{n}\right)^{T} \in R^{n}$ is the state vector of the system (2), $G_{i}, g_{i l}(i=1,2, \ldots, n ; l=1,2, \ldots, k)$ are nonlinear functions, and $q_{i l}(i=1,2, \ldots, n ; l=1,2, \ldots, k)$ are unknown parameters of the system (2).

Define the error between the drive and response systems as

$$
\begin{aligned}
e_{1} & =y_{1}-h_{1}\left(x_{1}, x_{2}, \ldots, x_{n}\right) x_{1}, \\
e_{2} & =y_{2}-h_{2}\left(x_{1}, x_{2}, \ldots, x_{n}\right) x_{2}, \\
& \vdots \\
e_{n} & =y_{n}-h_{n}\left(x_{1}, x_{2}, \ldots, x_{n}\right) x_{n}
\end{aligned}
$$

where the error vector is $e=\left(e_{1}, e_{2}, \ldots, e_{n}\right)^{T} ; h(x)=$ $\operatorname{diag}\left(h_{1}(x), h_{2}(x), \ldots, h_{n}(x)\right), h_{i}(x): R^{n} \rightarrow R(i=1,2, \ldots, n)$ are called scaling function factors, which compose the scaling function matrix $h(x)$.

And suppose $J=d(h(x) x) / d x=\operatorname{diag}\left(J_{1}, J_{2}, \ldots, J_{n}\right)$; the synchronization error can be obtained in the following compact form:

$$
e_{i}=G_{i}(y)+\sum_{l=1}^{k} q_{i l} g_{i l}(y)-J_{i} F_{i}(x)-J_{i} \sum_{j=1}^{m} p_{i j} f_{i j}(x)+u_{i} .
$$

We say that generalized function projective synchronization (GFPS) with respect to the scaling function matrix $h(x)$ is realized between the drive system (1) and response system (2) if a controller is designed such that the error system (4) can be achieved in the sense that

$$
\lim _{t \rightarrow \infty}\|e(t)\|=\lim _{t \rightarrow \infty}\|y(t)-h(x) x(t)\|=0 .
$$

From the definition of GFPS, we know that the synchronization between two different chaotic systems with unknown parameters is that a controller $U$ and a corresponding parameter update law are chosen to make the error system asymptotically converge to zero.

Remark 1. For the scaling function matrix $h(x)$, if $h_{1}(x)=$ $h_{2}(x)=\cdots=h_{n}(x)$, GFPS is simplified to FPS. If $h(x)=$ $h=\operatorname{diag}\left(h_{1}, h_{2}, \ldots, h_{n}\right)$ are all real constants, then FPS becomes PS. In particular, if $h(x)=\operatorname{diag}(-1,-1, \ldots,-1)$, FPS problem is antisynchronization, and if $h(x)=I=$ $\operatorname{diag}(1,1, \ldots, 1)$, FPS turns to complete synchronization. So we can flexibly choose the scaling function matrix $h(x)$ for the actual requirement in engineering.

\section{Design of the Adaptive Sliding Mode Controller}

As we know, all systems are inevitably influenced by exotic perturbations. For a chaotic system, small error may lead to serious deterioration of synchronic performance because of the Butterfly Effect. Among many control methods, sliding mode control is a robust control method which has many interesting features such as fast response, low sensitivity to 
external disturbances, robustness to the plant uncertainties, and easy realization. So, in this paper, we use sliding mode control method to design a controller to realize GFPS.

Generally speaking, sliding mode design involves two major stages; the first step is selecting a switching surface such that the sliding motion is asymptotically stable and has a prescribed performance. And, in this paper, we define switching surface as

$$
s_{i}=\lambda_{i} e_{i}, \quad(i=1,2, \ldots, n),
$$

where the sliding surface parameters $\lambda_{i}(i=1,2, \ldots, n)$ are positive constants.

Secondly, a controller is designed to guarantee that the system can reach and subsequently stay on the sliding surface. We adopt an adaptive sliding mode controller as

$$
\begin{aligned}
u_{i}= & J_{i} F_{i}(x)-G_{i}(y)-\xi_{i} \tanh \left(\eta_{i} s_{i}\right)+J_{i} \sum_{j=1}^{m} \widehat{p}_{i j} f_{i j}(x) \\
& -\sum_{l=1}^{k} \widehat{q}_{i l} g_{i l}(y), \quad(i=1,2, \ldots, n),
\end{aligned}
$$

where $\xi_{i}$ and $\eta_{i}$ are adaptation coefficients which tune the gain and steepness of the tanh function, respectively. The parameters $\widehat{p}_{i j}$ and $\widehat{q}_{i l}$ are estimates of parameter values $p_{i j}$ and $q_{i l}$. And their update laws are in the following:

$$
\begin{aligned}
\dot{\xi}_{i} & =-\alpha_{i}\left|s_{i}\right|\left|e_{i}\right|, \quad\left(\xi_{i}(0)=\xi_{i 0}\right) \\
\dot{\eta}_{i} & =-\beta_{i}\left|s_{i}\right|\left|e_{i}\right|, \quad\left(\eta_{i}(0)=\eta_{i 0}\right) \\
\dot{\vec{p}}_{i j} & =-\lambda_{i} J_{i} f_{i j} s_{i}, \quad(i=1,2, \ldots, n ; j=1,2, \ldots, m) \\
\widehat{q}_{i l} & =\lambda_{i} g_{i l} s_{i}, \quad(i=1,2, \ldots, n ; l=1,2, \ldots, k),
\end{aligned}
$$

where $\alpha_{i}, \beta_{i}, \xi_{i 0}$, and $\eta_{i 0}(i=1,2, \ldots, n)$ are all positive constants, and $\xi_{i 0}$ and $\eta_{i 0}$ are the initial values of $\xi_{i}$ and $\eta_{i}(i=$ $1,2, \ldots, n)$.

Theorem 2. For the given continuous differential scaling matrix function $h(x)$, if the parameters $\Psi_{i}(i=1,2, \ldots, n)$ are greater than or equal to zero, where $\Psi_{i}=\left(\lambda_{i} \xi_{i}\left|\tanh \left(\eta_{i} s_{i}\right)\right|+\right.$ $\left.\xi_{i} \alpha_{i}\left|e_{i}\right|+\eta_{i} \beta_{i}\left|e_{i}\right|\right)(i=1,2, \ldots, n)$, the error system will converge to the sliding surface $s(t)=0$ when one chooses the sliding mode controller (7) and the parameter update law (8).

Proof. Choose the Lyapunov function as

$$
\begin{aligned}
V=\frac{1}{2}( & \sum_{i=1}^{n} s_{i}^{2}+\sum_{i=1}^{n} \sum_{j=1}^{m}\left(\widehat{p}_{i j}-p_{i j}\right)^{2}+\sum_{i=1}^{n} \sum_{l=1}^{k}\left(\widehat{q}_{i l}-q_{i l}\right)^{2} \\
& \left.+\sum_{i=1}^{n} \xi_{i}^{2}+\sum_{i=1}^{n} \eta_{i}^{2}\right) .
\end{aligned}
$$

Taking derivative of the Lyapunov function with respect to time, one has

$$
\begin{aligned}
& \dot{V}=\sum_{i=1}^{n} s_{i} \dot{s}_{i}+\sum_{i=1}^{n} \sum_{j=1}^{m}\left(\widehat{p}_{i j}-p_{i j}\right) \dot{\hat{p}}_{i j}+\sum_{i=1}^{n} \sum_{l=1}^{k}\left(\widehat{q}_{i l}-q_{i l}\right) \dot{\hat{q}}_{i l} \\
& +\sum_{i=1}^{n} \xi_{i} \dot{\xi}_{i}+\sum_{i=1}^{n} \eta_{i} \dot{\eta}_{i} \\
& =\sum_{i=1}^{n} \lambda_{i} s_{i}\left(G_{i}(y)+\sum_{l=1}^{k} q_{i l} g_{i l}(y)-J_{i} F_{i}(x)\right. \\
& \left.-J_{i} \sum_{j=1}^{m} p_{i j} f_{i j}(x)+u_{i}\right) \\
& -\sum_{i=1}^{n} \sum_{j=1}^{m}\left(\widehat{p}_{i j}-p_{i j}\right)\left(\lambda_{i} J_{i} s_{i} f_{i j}(x)\right) \\
& +\sum_{i=1}^{n} \sum_{l=1}^{k}\left(\widehat{q}_{i l}-q_{i l}\right)\left(\lambda_{i} s_{i} g_{i l}(y)\right) \\
& -\sum_{i=1}^{n} \xi_{i} \alpha_{i}\left|s_{i}\right|\left|e_{i}\right|-\sum_{i=1}^{n} \eta_{i} \beta_{i}\left|s_{i}\right|\left|e_{i}\right| \\
& =\sum_{i=1}^{n} \lambda_{i} s_{i}\left(\sum_{l=1}^{k} q_{i l} g_{i l}(y)-J_{i} \sum_{j=1}^{m} p_{i j} f_{i j}(x)\right. \\
& \left.-\xi_{i} \tanh \left(\eta_{i} s_{i}\right)+J_{i} \sum_{j=1}^{m} \widehat{p}_{i j} f_{i j}(x)-\sum_{l=1}^{k} \widehat{q}_{i l} g_{i l}(y)\right) \\
& -\sum_{i=1}^{n} \sum_{j=1}^{m}\left(\widehat{p}_{i j}-p_{i j}\right)\left(\lambda_{i} J_{i} s_{i} f_{i j}(x)\right) \\
& +\sum_{i=1}^{n} \sum_{l=1}^{k}\left(\widehat{q}_{i l}-q_{i l}\right)\left(\lambda_{i} s_{i} g_{i l}(y)\right) \\
& -\sum_{i=1}^{n} \xi_{i} \alpha_{i}\left|s_{i}\right|\left|e_{i}\right|-\sum_{i=1}^{n} \eta_{i} \beta_{i}\left|s_{i}\right|\left|e_{i}\right| \\
& =-\sum_{i=1}^{n} \lambda_{i} s_{i} \xi_{i} \tanh \left(\eta_{i} s_{i}\right)-\sum_{i=1}^{n} \xi_{i} \alpha_{i}\left|s_{i}\right|\left|e_{i}\right|-\sum_{i=1}^{n} \eta_{i} \beta_{i}\left|s_{i}\right|\left|e_{i}\right| \\
& \leq-\sum_{i=1}^{n}\left(\lambda_{i} \xi_{i}\left|\tanh \left(\eta_{i} s_{i}\right)\right|+\xi_{i} \alpha_{i}\left|e_{i}\right|+\eta_{i} \beta_{i}\left|e_{i}\right|\right)\left|s_{i}\right| \\
& =-\sum_{i=1}^{n} \Psi_{i}\left|s_{i}\right|
\end{aligned}
$$

where $\Psi_{i}=\left(\lambda_{i} \xi_{i}\left|\tanh \left(\eta_{i} s_{i}\right)\right|+\xi_{i} \alpha_{i}\left|e_{i}\right|+\eta_{i} \beta_{i}\left|e_{i}\right|\right)(i=$ $1,2, \ldots, n)$

For a given error system, if we deduce that $\Psi_{i}$ is greater than or equal to zero, that is, $\left(\lambda_{i} \xi_{i}\left|\tanh \left(\eta_{i} s_{i}\right)\right|+\xi_{i} \alpha_{i}\left|e_{i}\right|+\right.$ $\left.\eta_{i} \beta_{i}\left|e_{i}\right|\right) \geq 0(i=1,2, \ldots, n)$, the following conclusions will be derived:

$$
\dot{V} \leq-\sum_{i=1}^{n} \Psi_{i}\left|s_{i}\right|=-\omega(t) \leq 0
$$


where $\omega(t)=\sum_{i=1}^{n} \Psi_{i}\left|s_{i}\right|$. Integrating (11) from zero to $t$ yields

$$
V(0) \geq V(t)+\int_{0}^{t} \omega(s) d s \geq \int_{0}^{t} \omega(s) d s,
$$

as $t$ approach infinite, and $V(0)$ is positive and bounded, so $\lim _{t \rightarrow \infty} \int_{0}^{t} \omega(s) d s$ exits and is finite. By Barbalat lemma, we have

$$
\lim _{t \rightarrow \infty} \omega(t)=\lim _{t \rightarrow \infty} \sum_{i=1}^{n} \Psi_{i}\left|s_{i}\right|=0 .
$$

Now, one will ask, "how can we conclude $\Psi_{i} \geq 0$ ( $i=$ $1,2, \ldots, n)$ ?" and from theirs expressions, we can see that it is hard to conclude $\Psi_{i} \geq 0(i=1,2, \ldots, n)$ by means of formula derivation. But we can get it with computer simulation, which will be seen in the following concrete example.

If the parameters $\Psi_{i}(i=1,2, \ldots, n)$ are greater than or equal to zero, the sliding mode controller and the parameter update law are chosen by (7) and (8). this concludes that $s_{i}(i=1,2, \ldots, n)$ are equal to zero when the system is operating on the sliding surface, and (6) is satisfied, so GFPS of the drive system (1) and response system (2) can be achieved as time goes on.

Remark 3. As we know, the conventional sliding mode controllers often use the sign function [17, 18], but the discontinuity of the sign function causes the chattering. In order to avoid the chattering, the discontinuous sign function is replaced by the continuous tanh function with the adaptive gain and steepness. As in $[15,16]$, the function is used as an approximate of the sign function.

\section{Numerical Simulations}

In this section, we take two different systems as the drive and response systems, respectively, to validate the robustness and effectiveness of the proposed method.

The drive system is the Lorenz system, which is the following mathematical expression:

$$
\begin{gathered}
\dot{x}_{1}=p_{1}\left(x_{2}-x_{1}\right), \\
\dot{x}_{2}=p_{2} x_{1}-x_{1} x_{3}-x_{2}, \\
\dot{x}_{3}=-p_{3} x_{3}+x_{1} x_{2},
\end{gathered}
$$

where $x=\left(x_{1}, x_{2}, x_{3}\right)^{T}$ is the state vector of the system (14), and $p=\left(p_{1}, p_{2}, p_{3}\right)^{T}$ is the parameter vector of (14). When these parameters are taken as $p_{1}=10, p_{2}=28$, and $p_{3}=8 / 3$, the system is chaotic.

The Genesio-Tesi (GT) system is chosen as the response system, and the expression with a controller is

$$
\begin{gathered}
\dot{y}_{1}=y_{2}+u_{1}, \\
\dot{y}_{2}=y_{3}+u_{2}, \\
\dot{y}_{3}=-q_{1} y_{1}-q_{2} y_{2}-q_{3} y_{3}+y_{1}^{2}+u_{3},
\end{gathered}
$$

where $y=\left(y_{1}, y_{2}, y_{3}\right)^{T}$ is the state vector of the system (15) and $q=\left(q_{1}, q_{2}, q_{3}\right)^{T}$ is the parameter vector of (15). When these parameters are taken as $q_{1}=1.0, q_{2}=1.1$, and $q_{3}=$ 0.44 , the system is chaotic.

In numerical simulation, the scaling function matrix is chosen as

$$
\begin{aligned}
& h_{1}\left(x_{1}\right)=v_{11} \sin \left(v_{12} x_{1}+v_{13}\right)+v_{14}, \\
& h_{2}\left(x_{2}\right)=v_{21} \sin \left(v_{22} x_{2}+v_{23}\right)+v_{24}, \\
& h_{3}\left(x_{3}\right)=v_{31} \sin \left(v_{32} x_{3}+v_{33}\right)+v_{34},
\end{aligned}
$$

and then the derivative of $h_{i}(i=1,2,3)$ can be derived as

$$
\begin{aligned}
& J_{1}=v_{11} \sin \left(v_{12} x_{1}+v_{13}\right)+v_{11} v_{12} x_{1} \cos \left(v_{12} x_{1}+v_{13}\right)+v_{14}, \\
& J_{2}=v_{21} \sin \left(v_{22} x_{2}+v_{23}\right)+v_{21} v_{22} x_{2} \cos \left(v_{22} x_{2}+v_{23}\right)+v_{24}, \\
& J_{3}=v_{31} \sin \left(v_{32} x_{3}+v_{33}\right)+v_{31} v_{32} x_{3} \cos \left(v_{32} x_{3}+v_{33}\right)+v_{34},
\end{aligned}
$$

where the parameters $v_{11}=0.05, v_{12}=0.1, v_{13}=-0.2, v_{14}=$ $1.0, v_{21}=0.02, v_{22}=-0.2, v_{23}=0.3, v_{24}=1.0, v_{31}=0.03$, $v_{32}=0.3, v_{33}=-0.5$, and $v_{34}=1.0$.

Now, the parameters $\Psi_{1}, \Psi_{2}$, and $\Psi_{3}$ of the error system are firstly simulated with the help of the computer. The sliding surface parameters $\lambda_{i}(i=1,2, \ldots, n)$ are all selected as 1.5. Vectors $[3,-4,2.6]$ and $[0.1,0.3,0]$ are selected as the initial conditions of the Lorenz and GT systems, respectively. And vectors $[8,10,5]$ and $[0.6,0.7,10]$ are selected as the initial values of the adaptation vector parameters $\widehat{p}$ and $\widehat{q}$. And the initial values of adaptation coefficients are chosen as $\xi_{0}=$ $[10,10,10]$ and $\eta_{0}=[0.01,0.01,0.01]$. The corresponding parameters $\alpha_{i}, \beta_{i}(i=1,2,3)$ are chosen as $\alpha_{1}=1.0, \alpha_{2}=1.1$, $\alpha_{3}=0.8, \beta_{1}=0.8, \beta_{2}=0.67$, and $\beta_{3}=0.78$.

Figure 1 gives time evolution of the parameters $\Psi_{1}, \Psi_{2}$, and $\Psi_{3}$ of the error system. It shows that $\Psi_{1}, \Psi_{2}$, and $\Psi_{3}$ are greater than zero in the first few seconds and $\Psi_{1}, \Psi_{2}$, and $\Psi_{3}$ become zero rapidly as time goes on. According to Theorem 2, the control inputs are obtained by (7)

$$
\begin{gathered}
u_{1}=-y_{2}-\xi_{1} \tanh \left(\eta_{1} s_{1}\right)+J_{1} \widehat{p}_{1}\left(x_{2}-x_{1}\right), \\
u_{2}=-y_{3}-\xi_{2} \tanh \left(\eta_{2} s_{2}\right)-J_{2}\left(x_{1} x_{3}+x_{2}\right)+J_{2} \widehat{p}_{2} x_{1}, \\
u_{3}=-y_{1}^{2}+J_{3} x_{1} x_{2}-\xi_{3} \tanh \left(\eta_{3} s_{3}\right)+\widehat{q}_{1} y_{1}+\widehat{q}_{2} y_{2} \\
+\widehat{q}_{3} y_{3}-J_{3} \widehat{p}_{3} x_{3},
\end{gathered}
$$

where the parameter $\widehat{p}=\left(\widehat{p}_{1}, \widehat{p}_{2}, \widehat{p}_{3}\right)^{T}$ and $\hat{q}=\left(\widehat{q}_{1}, \widehat{q}_{2}, \widehat{q}_{3}\right)^{T}$; their update laws can be obtained by (8). Thus, GFPS of the Lorenz system (14) and response system (15) can be realized.

Figure 2 shows time evolution of GFPS errors of Lorenz and GT systems, without exotic perturbation. We can see that, with the adaptive sliding mode controller (18), the GFPS errors converge to zero rapidly, which verifies that our proposed method is feasible. Further, Figure 3 gives time evolution of the controller, $u_{1}, u_{2}$, and $u_{3}$. It shows that the chattering phenomenon does not really occur by the use of the continuous tanh function with the adaptive gain and steepness. 


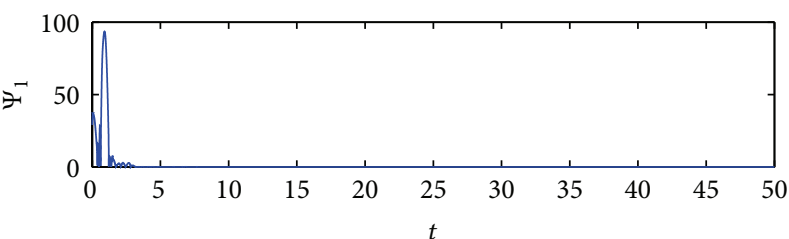

(a)

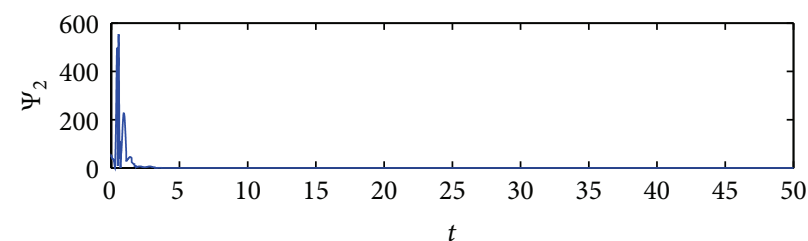

(b)

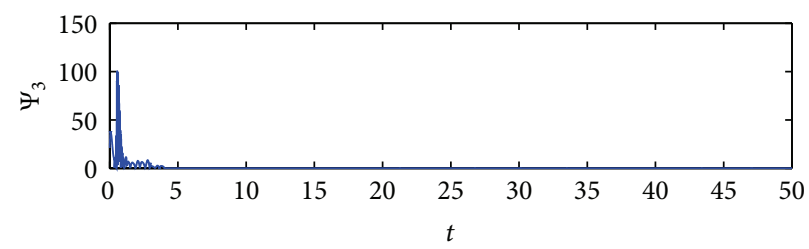

(c)

FIGURE 1: Time evolution of the parameters $\Psi_{1}, \Psi_{2}$, and $\Psi_{3}$ of the error system.

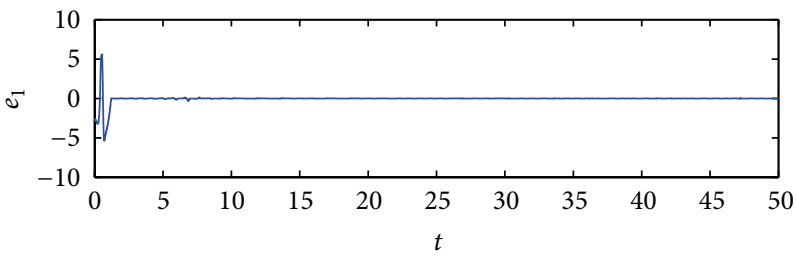

(a)

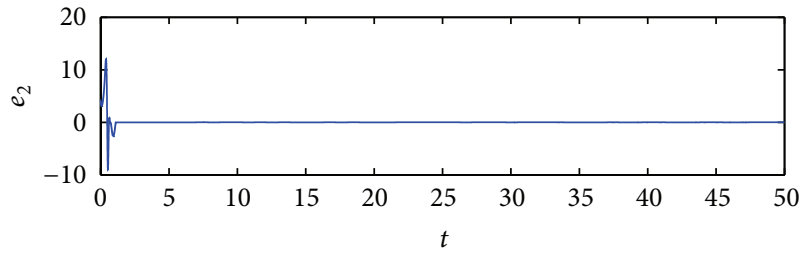

(b)

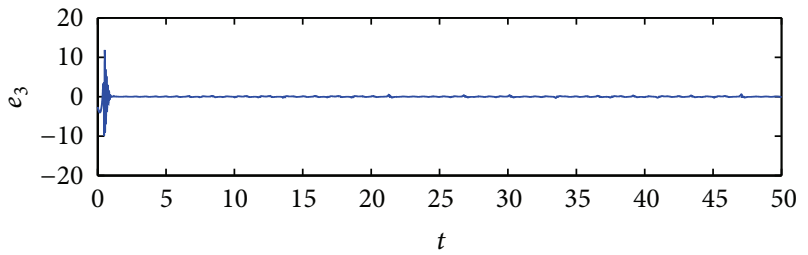

(c)

FIGURE 2: Time evolution of GFPS errors of Lorenz and GT system without exotic perturbation.

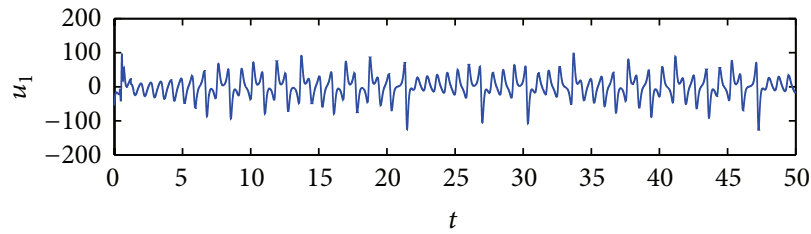

(a)

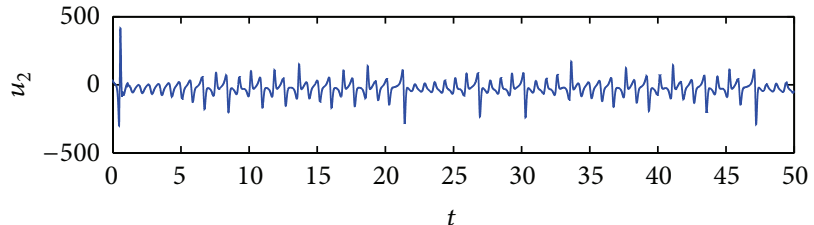

(b)

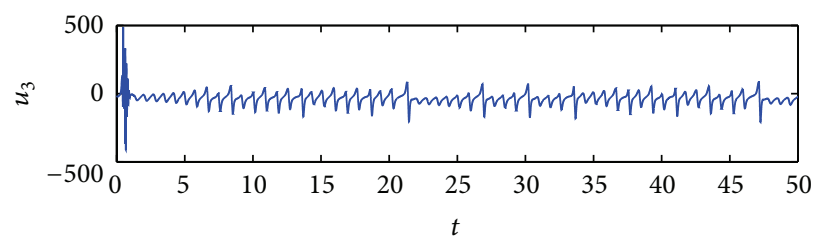

(c)

FIgURE 3: Time evolution of the controller $u_{1}, u_{2}$, and $u_{3}$. 


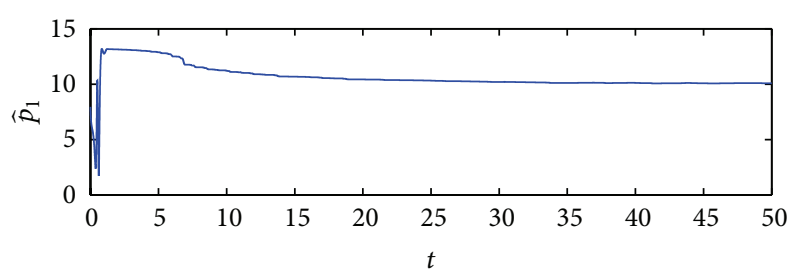

(a)

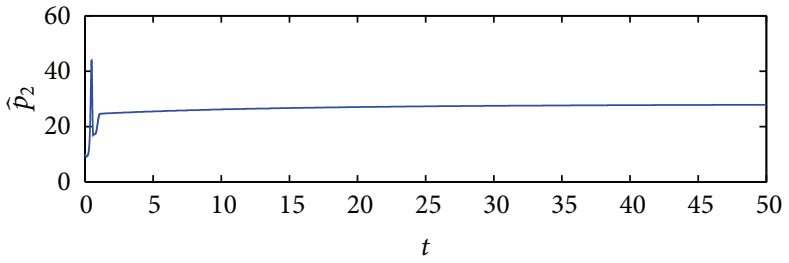

(b)

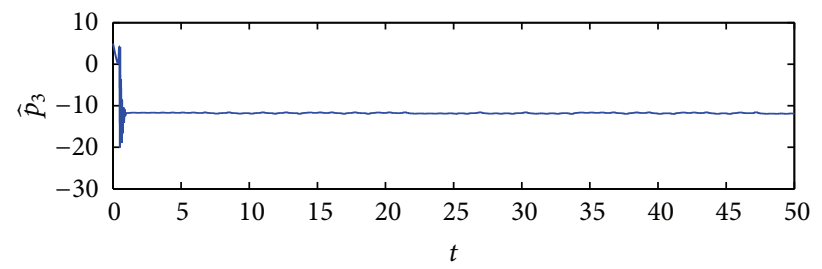

(c)

FIgURE 4: Time evolution of the parameters $\widehat{p}_{1}, \widehat{p}_{2}$, and $\widehat{p}_{3}$ of the error system.

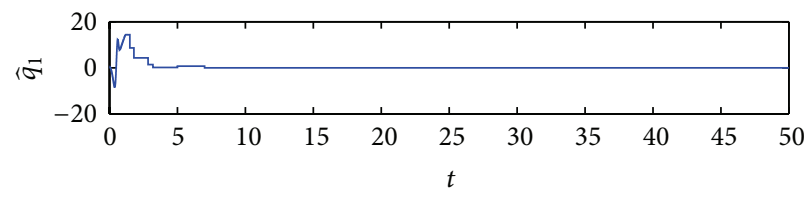

(a)

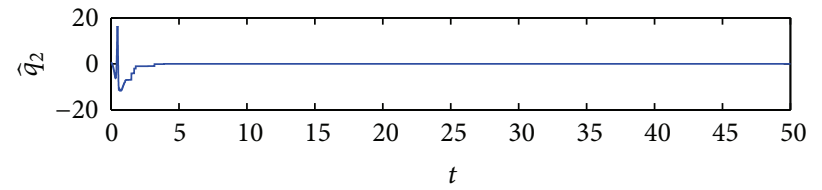

(b)

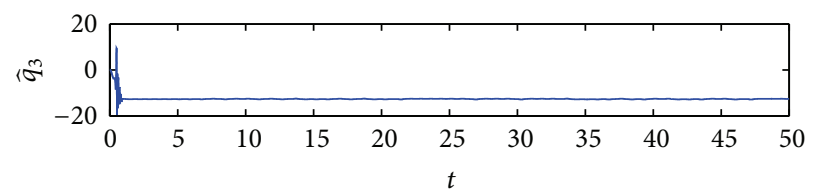

(c)

FIGURE 5: Time evolution of the parameters $\widehat{q}_{1}, \widehat{q}_{2}$, and $\widehat{q}_{3}$ of the error system.

Figures 4 and 5 illustrate time evolution of the parameters $\widehat{p}_{1}, \widehat{p}_{2}, \widehat{p}_{3}, \widehat{p}_{3}, \widehat{q}_{1}, \widehat{q}_{2}$, and $\widehat{q}_{3}$ of the error system. They show that these parameters do not converge to the parameters $p_{1}=$ $10, p_{2}=28, p_{3}=8 / 3, q_{1}=1.0, q_{2}=1.1$, and $q_{3}=0.44$ of the drive (14) and response systems (15) as time $t$ goes to infinity. So the unknown parameters cannot be estimated accurately by means of the parameters $\widehat{p}_{1}, \widehat{p}_{2}, \widehat{p}_{3}, \widehat{p}_{3}, \widehat{q}_{1}, \widehat{q}_{2}$, and $\widehat{q}_{3}$. In addition, we can derive from (8) that the update laws of $\hat{p}$ and $\widehat{q}$ will converge to zero when the error signals tend to zero, so, as time goes on, the parameters $\widehat{p}$ and $\widehat{q}$ converge to some constant and do not necessarily tend to $p$ and $q$.

Figure 6 gives time evolution of the controlled signals $y_{1}$, $y_{2}$, and $y_{3}$ of the response system (15). It illustrates that, with the controller, the response system is still chaotic.

To further verify the robustness of the controller, it is assumed that the drive and response systems are influenced by exotic perturbations. It is assumed that the state vector of the system (14) is perturbed by $\delta_{1}[\sin 5 t, \cos 7 t, \sin 4 t]$ and the state vector of the system (15) is perturbed by $\delta_{2}[\cos 2 t, \sin 6 t, \sin 5 t]$, where $\delta_{1}$ and $\delta_{2}$ are the amplitudes of the exotic perturbations. When $\delta_{1}=\delta_{2}=0$, that is, the systems (14) and (15) are all undisturbed by external factors, from Figure 1, we still can realize GFPS.

Further, the amplitudes are selected as $\delta_{1}=\delta_{2}=1.0$. Figure 7 shows time evolution of GFPS errors of Lorenz and GT systems with the amplitude 1.0. We can see that the GFPS errors converge to zero rapidly, which implies our proposed method is robust. Secondly, the amplitudes are selected as $\delta_{1}=\delta_{2}=2.5$. Figure 8 shows time evolution of GFPS errors of Lorenz and GT systems with the amplitude 2.5.

It shows that the GFPS errors $e_{1}$ and $e_{2}$ still converge to zero and $e_{3}$ slightly fluctuates around zero. Figure 9 gives time evolution of GFPS errors of Lorenz and GT systems with the amplitude 3.0. It implies that the GFPS errors $e_{1}$ and $e_{2}$ still converge to zero and $e_{3}$ fluctuates around zero. The fluctuation of $e_{3}$ around zero in Figure 9 becomes a little greater than that in Figure 8. Figure 10 illustrates a comparison of time evolution of the error $e_{3}$ with different amplitudes, which further show the greater amplitude of the exotic perturbation, the greater fluctuation around zero of the GFPS errors. 


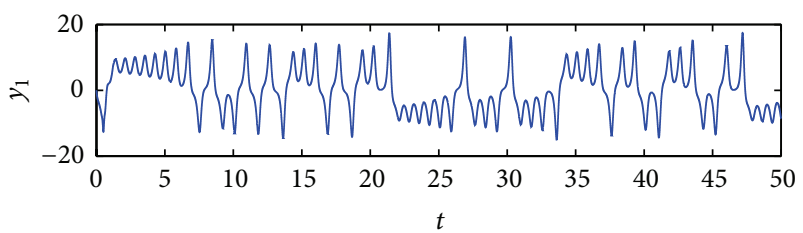

(a)

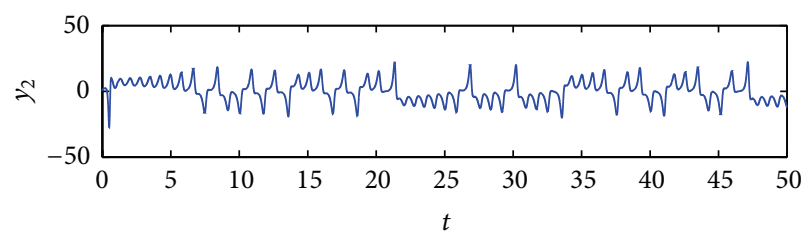

(b)

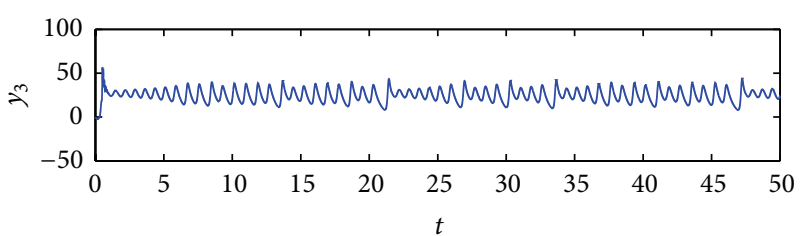

(c)

FIGURE 6: Time evolution of the controlled signals $y_{1}, y_{2}$, and $y_{3}$ of the response system (15).

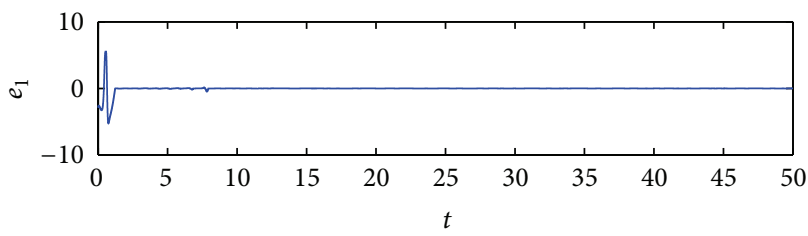

(a)

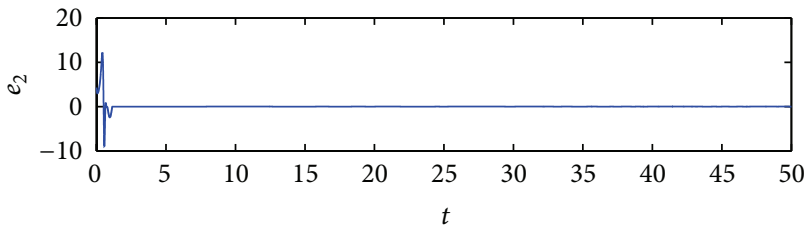

(b)

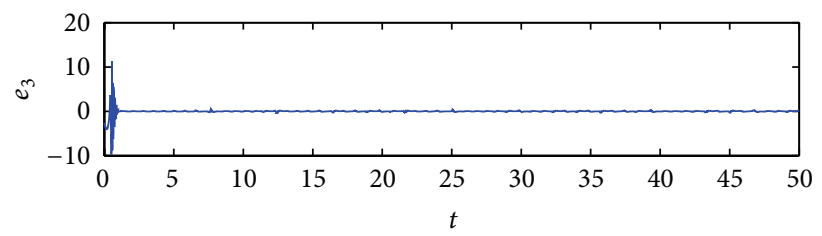

(c)

FIGURE 7: Time evolution of GFPS errors of Lorenz and GT system with the amplitude 1.0.

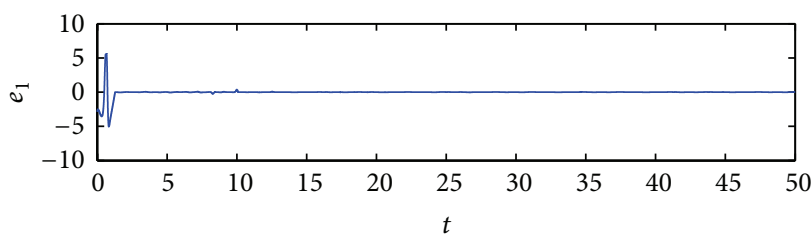

(a)

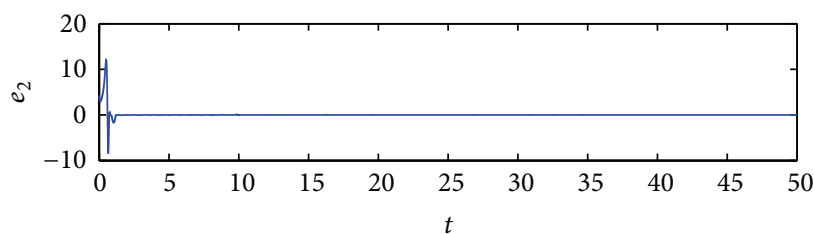

(b)

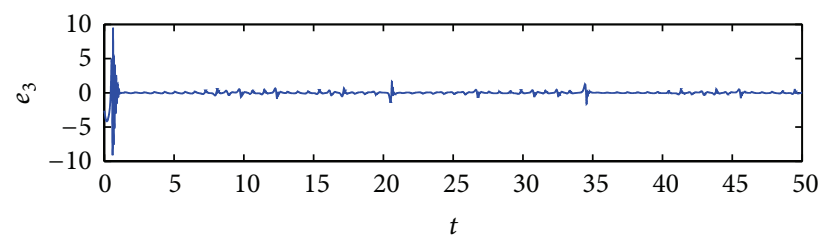

(c)

FIGURE 8: Time evolution of GFPS errors of Lorenz and GT system with the amplitude 2.5. 


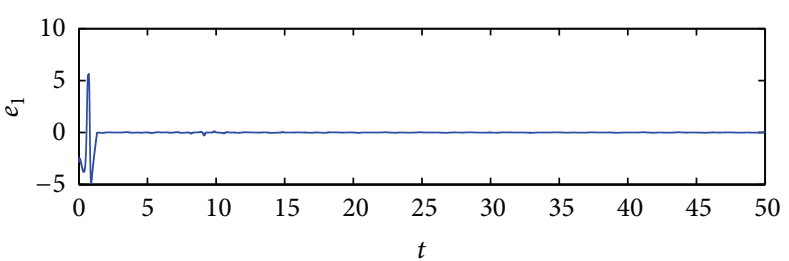

(a)

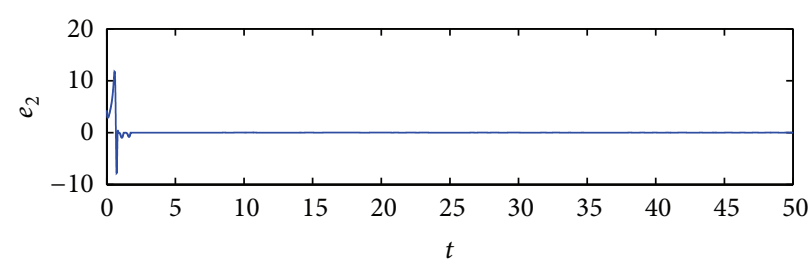

(b)

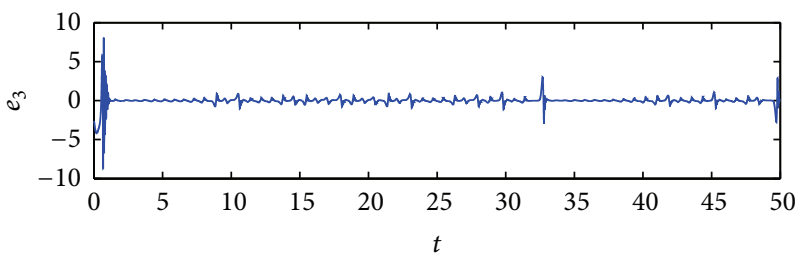

(c)

FIgURE 9: Time evolution of GFPS errors of Lorenz and GT system with the amplitude 3.0.

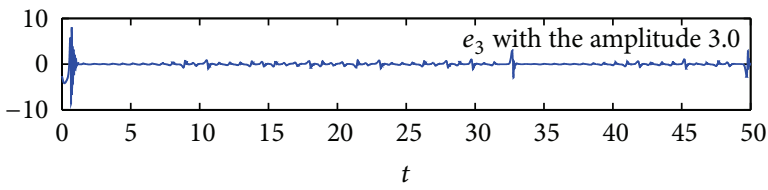

(a)

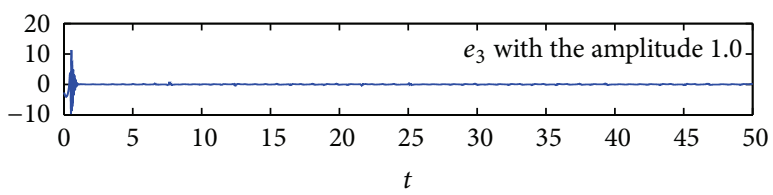

(c)

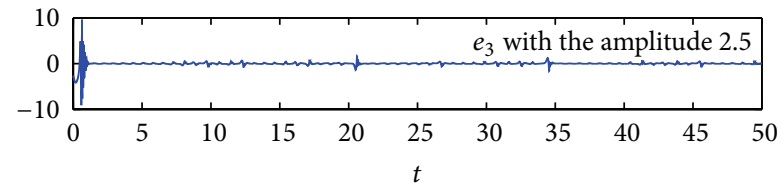

(b)

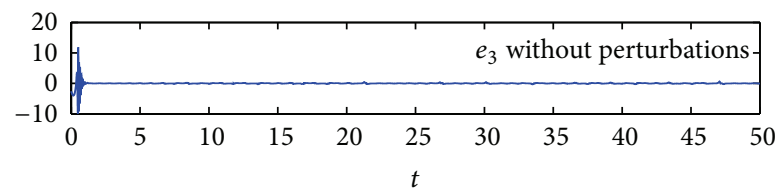

(d)

Figure 10: Time evolution of the error $e_{3}$ with different amplitude.

All these results imply that our proposed method is strongly robust to exotic perturbations, especially the errors $e_{1}$ and $e_{2}$; they still converge to zero when the amplitude of the exotic perturbation is 3 . So the method is effective.

\section{Conclusions}

Because the sliding mode control has some interesting features such as fast response, low sensitivity to external disturbances, robustness to the plant uncertainties, and easy realization, in this paper, we use this method to realize generalized function projective synchronization of two different chaotic systems with unknown parameters. According to two major stages of sliding mode design, we give the sliding mode surface and adaptive sliding controller. Finally, numerical simulations of two different chaotic systems are presented to show that the proposed method is effective and robust to exotic perturbations even when both drive and response systems are perturbed with external disturbances.

\section{Acknowledgment}

This Project is supported by the National Natural Science Foundation of China (Grant nos. 11002114, 60901076, 11102157, 11202035 , and 11272258).

\section{References}

[1] L. M. Pecora and T. L. Carroll, "Synchronization in chaotic systems," Physical Review Letters, vol. 64, no. 8, pp. 821-824, 1990.

[2] F. Wang and C. Liu, "A new criterion for chaos and hyperchaos synchronization using linear feedback control," Physics Letters A, vol. 360, no. 2, pp. 274-278, 2006.

[3] X. Wu, G. Chen, and J. Cai, "Chaos synchronization of the master-slave generalized Lorenz systems via linear state error feedback control," Physica D, vol. 229, no. 1, pp. 52-80, 2007.

[4] H.-H. Chen, G.-J. Sheu, Y.-L. Lin, and C.-S. Chen, "Chaos synchronization between two different chaotic systems via nonlinear feedback control," Nonlinear Analysis. Theory, Methods \& Applications A, vol. 70, no. 12, pp. 4393-4401, 2009. 
[5] D. B. Huang, "Simple adaptive-feedback controller for identical chaos synchronization," Physical Review E, vol. 71, Article ID 037203, 2005.

[6] Z.-M. Ge and C.-H. Yang, "Pragmatical generalized synchronization of chaotic systems with uncertain parameters by adaptive control," Physica D, vol. 231, no. 2, pp. 87-94, 2007.

[7] D. Zhang and J. Xu, "Projective synchronization of different chaotic time-delayed neural networks based on integral sliding mode controller," Applied Mathematics and Computation, vol. 217, no. 1, pp. 164-174, 2010.

[8] N. Cai, Y. Jing, and S. Zhang, "Modified projective synchronization of chaotic systems with disturbances via active sliding mode control," Communications in Nonlinear Science and Numerical Simulation, vol. 15, no. 6, pp. 1613-1620, 2010.

[9] Y. Wang, X. Xu, L. Dai, and B. Hou, "Synchronization of a class of partially unknown chaotic systems with integral observer," in Proceedings of the 36th Annual Conference of the IEEE Industrial Electronics Society (IECON '10), pp. 231-235, November 2010.

[10] R. Mainieri and J. Rehacek, "Projective synchronization in three-dimensional chaotic systems," Physical Review Letters, vol. 82, no. 15, pp. 3042-3045, 1999.

[11] Y. Chen, H. An, and Z. Li, "The function cascade synchronization approach with uncertain parameters or not for hyperchaotic systems," Applied Mathematics and Computation, vol. 197, no. 1, pp. 96-110, 2008.

[12] X.-Y. Wang and B. Fan, "Generalized projective synchronization of a class of hyperchaotic systems based on state observer," Communications in Nonlinear Science and Numerical Simulation, vol. 17, no. 2, pp. 953-963, 2012.

[13] Z. Li and X. Zhao, "Generalized function projective synchronization of two different hyperchaotic systems with unknown parameters," Nonlinear Analysis. Real World Applications, vol. 12, no. 5, pp. 2607-2615, 2011.

[14] C.-C. Yang and C.-J. Ou, "Adaptive terminal sliding mode control subject to input nonlinearity for synchronization of chaotic gyros," Communications in Nonlinear Science and Numerical Simulation, vol. 18, no. 3, pp. 682-691, 2013.

[15] W. Xiang and F. Chen, "Robust synchronization of a class of chaotic systems with disturbance estimation," Communications in Nonlinear Science and Numerical Simulation, vol. 16, no. 8, pp. 2970-2977, 2011.

[16] M. P. Aghababa and M. E. Akbari, "A chattering-free robust adaptive sliding mode controller for synchronization of two different chaotic systems with unknown uncertainties and external disturbances," Applied Mathematics and Computation, vol. 218, no. 9, pp. 5757-5768, 2012.

[17] X. C. Li, W. Xu, and Y. Z. Xiao, "Adaptive slide mode control for a class of chaotic systems with perturbations," Acta Physica Sinica. Wuli Xuebao, vol. 57, no. 8, pp. 4721-4728, 2008.

[18] C.-C. Yang, "Robust synchronization and anti-synchronization of identical $\Phi_{6}$ oscillators via adaptive sliding mode control," Journal of Sound and Vibration, vol. 331, no. 3, pp. 501-509, 2012. 


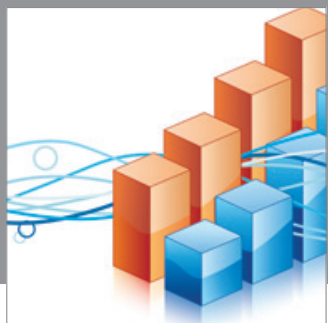

Advances in

Operations Research

mansans

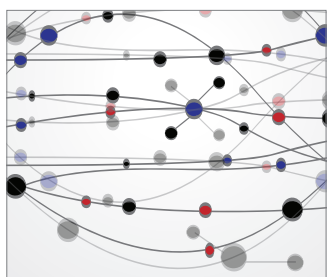

The Scientific World Journal
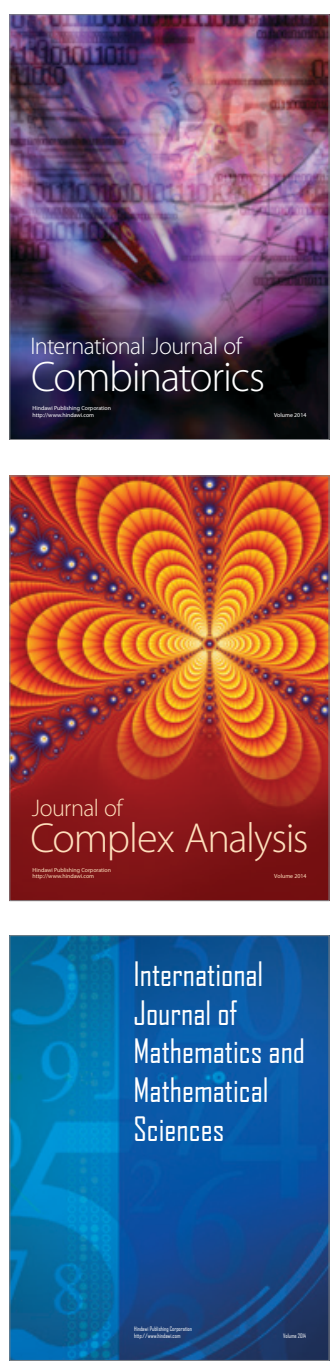
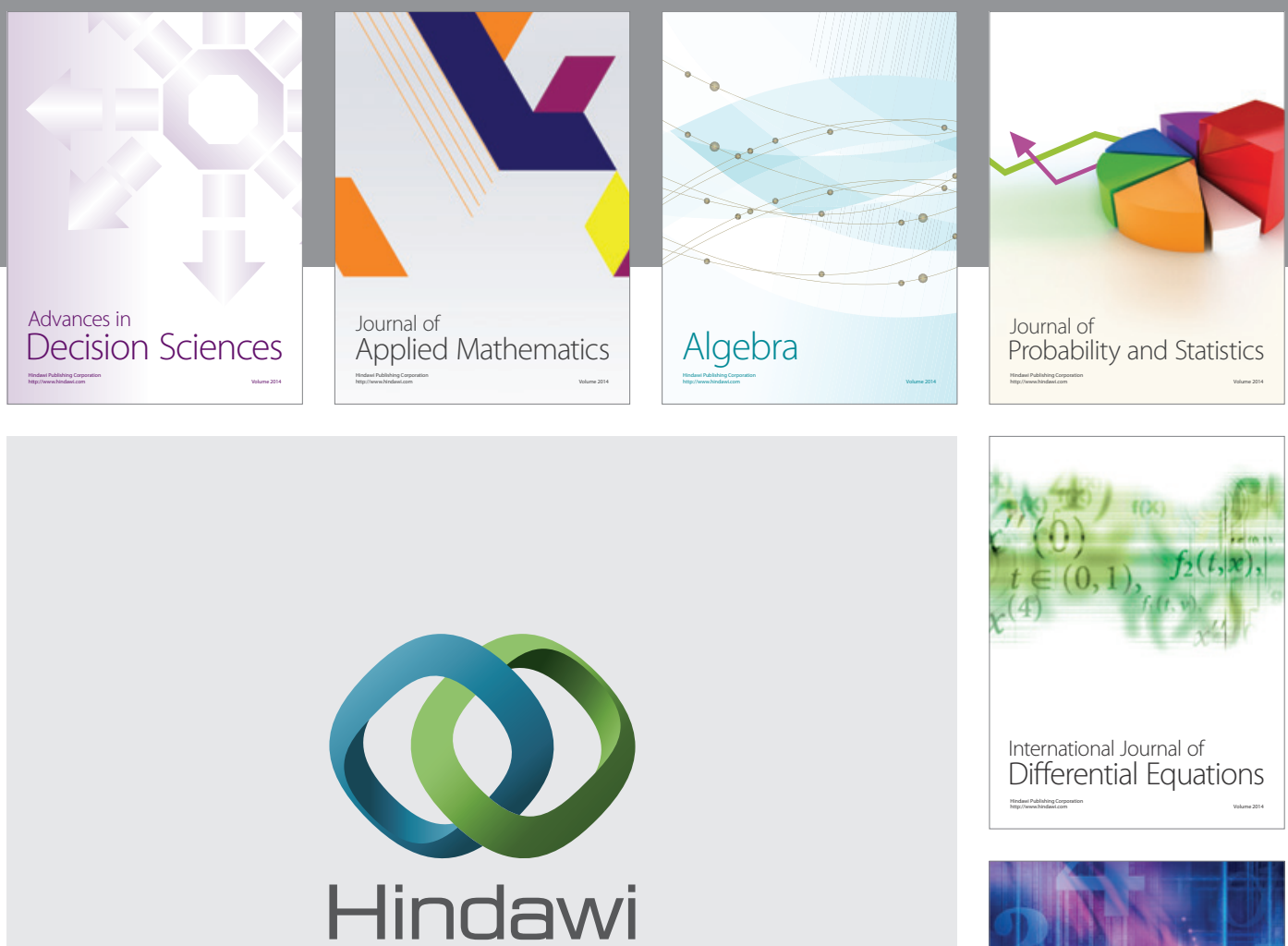

Submit your manuscripts at http://www.hindawi.com
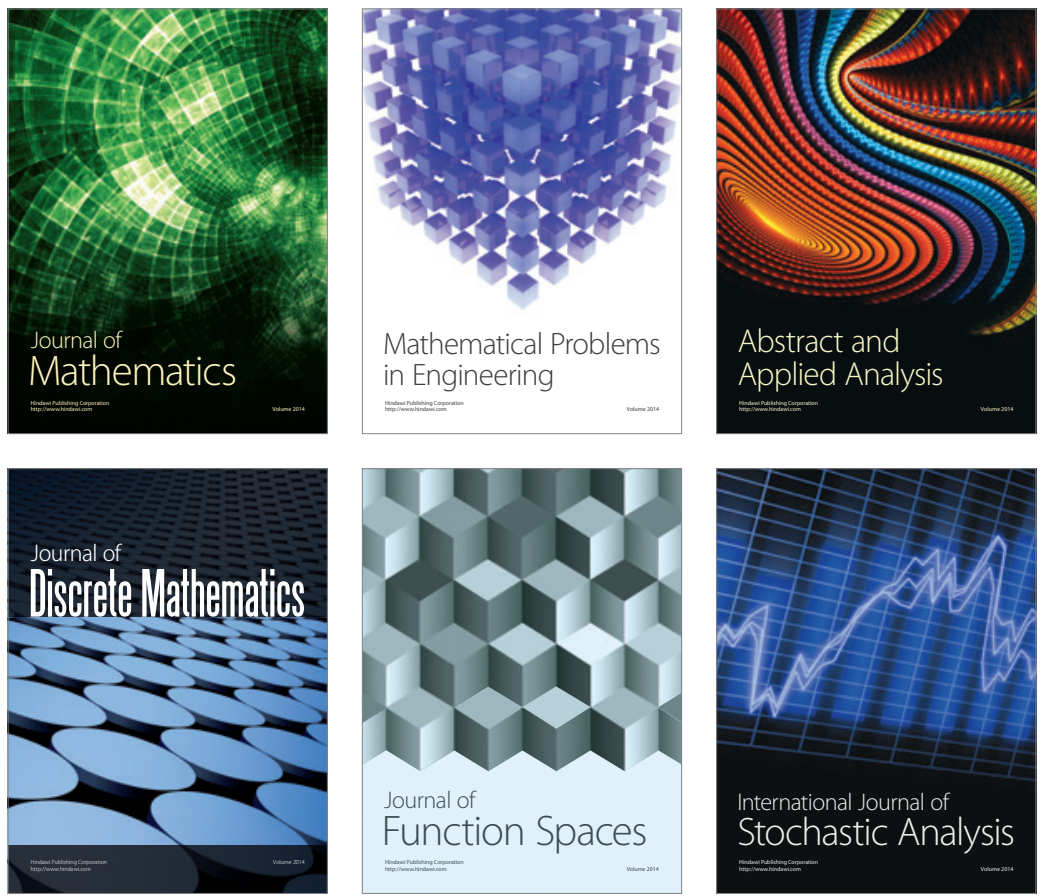

Journal of

Function Spaces

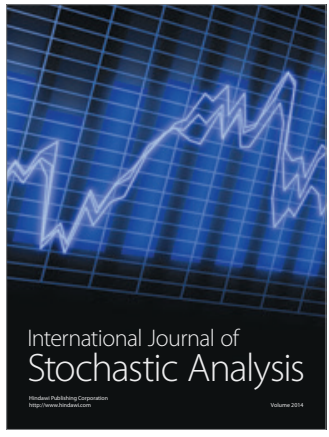

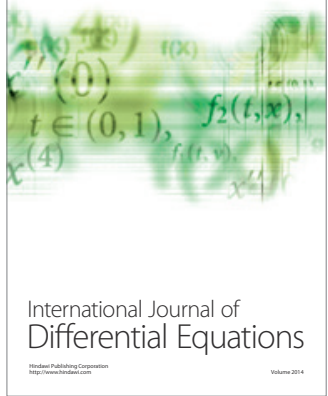
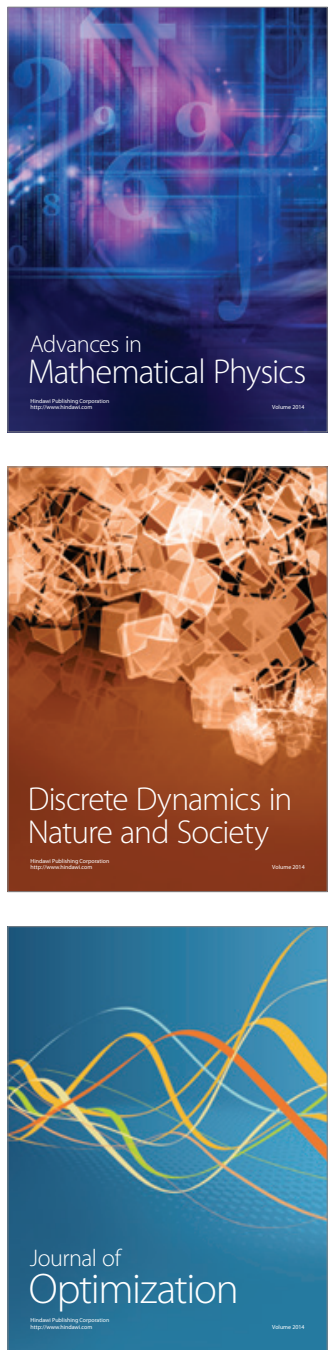